\title{
Expected levels of behaviour problems in a population sample of children between four and eighteen years
}

Bongers I, Koot H, Ende J et al. The normative development of child and adolescent problem behavior.J Abnorm Psychol 2003;112:179-192.

\author{
QUESTION: What is the developmental trajectory of the Child Behavior Checklist in \\ children in the general population?
}

\section{Design}

Longitudinal study of a single population-based cohort.

\section{Setting}

Zuid-Holland province, The Netherlands; 1993-1991.

\section{Participants}

2076 children aged 4-16 years in 1983, randomly sampled from the general population. Participants were followed up in 1985, 1987, 1989 and 1991.

\section{Main outcome measures}

Child behaviour checklist scores at each follow up interval for each of the following components (internalising scale: scoring on feelings of anxiety/depression, withdrawal, somatic complaints; externalising scale: scoring on aggressive behaviour, delinquent behaviour; and scales scoring on attention problems, thought problems and social problems). Individual scores were added to obtain a total problems score. Trajectories were analysed by gender and age.

\section{Main results}

Internalising problems were similar in girls and boys in childhood, but more girls than boys were affected in adolescence. Externalising problems were more common in boys than girls, and decreased with age. Attention problems increased for both boys and girls (more so for boys than girls) until age 11, and then decreased. The total problems score decreased with age for girls and boys.

\section{Conclusions}

The results reflect a normal reference trajectory for behaviour problems in the general population. However, these results are based on parent-reported symptoms, which may not accurately assess internalising problems and behaviours.

\section{COMMENTARY}

Bongers et al examine changes in levels of problem behaviour, or normative developmental trajectories, of children and adolescents aged 4-18 years. They report that most behavioural syndromes change with age, and trajectories differ for boys versus girls.

Can we apply the results of this study more broadly? Yes. First, findings from this general population sample of 2076 Dutch children are generalisable to similar samples of children and adolescents. Second, the measurement tool, the Child Behavior Checklist (CBCL), is widely used, standardised, and generalisable across cultures. ${ }^{2}$ Finally, the use of growth curve analyses allows use of data representative of broader populations as this technique overcomes a major short-coming of traditional approaches to analysis of multi-wave data, where all participants must have data at all time points. ${ }^{3}$

Can we utilise the results of this study clinically? To some extent. These results can inform any individual who works with children, inside or outside of clinical settings, about what to expect as "typical" development of child and adolescent problem behaviours. When evaluating children or adolescents in specialised mental health clinics, an understanding of the normative development of problem behaviours is an important basis for evaluating psychopathology. However, CBCL and DSM diagnostic categories are not identical, and while general problem behaviours may suggest broad interventions, knowledge of evidence-based interventions for specific psychiatric disorders is critical to clinical practice.

Can we learn more about normative development of problem behaviours? Yes. Other longitudinal data sets with general population multiple birth cohort samples of children of similar ages can inform us further (e.g., Canadian National Longitudinal Survey of Children and Youth). ${ }^{4}$ Analytic techniques that are responsive to issues of sample maintenance are available. Data from multiple informants can further broaden our understanding of the normative development of problem behaviours in children and adolescents.

Ellen L Lipman, MD FRCPC Associate Professor

Department of Psychiatry and Behavioural Neurosciences Faculty of Health Sciences McMaster University and Hamilton Health Sciences Canada

1 Achenbach TM. Manual for the Child Behavior Checklist/ 4-18 and 1991 Child Profile. Burlingtom: University of Vermont, Department of Psychiatry, 1991.

2 De Groot A, Koot HM, Verhulst FC. Cross-cultural generalizabilitiy of the Child Behavior Checklist cross-informant syndromes. Psycholog Assess 1994; 6: 225-30.

3 Francis DJ, Fletcher JM, Stuebing KK, et al. Analysis of change: modeling individual growth. J Consult Clin Psycholog 1991; 59: 27-37.

4 Special Surveys Division. National longitudinal survey of children and youth: user's handbook and microdata guide. Ottawa: Statistics Canada and Human Resources Development Canada, 1996.
This work was funded by the Sophia Scientific Foundation.

For correspondence: I Bongers. Department of Child and Adolescent Psychiatry. Erasmus MC-Sophia Children's Hospital, Dr.

Molewaterplein 60, 3015 GJ Rotterdam the Netherlands. E-mail:

i.l.bongers@ erasmusmc.nl 